\title{
De novo synthesis of glial glutamate and glutamine in young mice requires aspartate provided by the neuronal mitochondrial aspartate-glutamate carrier aralar/AGC1
}

\author{
Beatriz Pardo*, Laura Contreras and Jorgina Satrústegui \\ Departamento de Biología Molecular, Centro de Biología Molecular Severo Ochoa UAM-CSIC, CIBER de Enfermedades Raras (CIBERER), Universidad Autónoma de \\ Madrid, Madrid, Spain \\ *Correspondence: bpardo@cbm.uam.es
}

Edited by:

Leif Hertz, China Medical University, China

Keywords: AGC1, aralar, aspartate, glial glutamine, mitochondrial aspartate-glutamate carrier

\section{INTRODUCTION}

In brain, the glutamate-glutamine and the glutamate-glutamine-GABA cycles are essential for efficient glutamatergic and GABAergic neurotransmission. The interactions between neurons and astrocytes required for the operation of these cycles have received considerable attention since their discovery (1). In gray matter, glutamate released from glutamatergic neurons is mostly taken up into astrocytes $(2,3)$ where it is converted into glutamine which is sent back to the neurons for conversion to glutamate. This drain of glutamate released from neurons to astrocytes is compensated for by a flow of glutamine from astrocytes to neurons, thus closing the glutamateglutamine cycle.

In addition to transcellular cycling, the glutamate-glutamine cycle faces losses of components, mainly glutamate, by oxidation, which is balanced by the anaplerotic synthesis of glutamate and glutamine in astrocytes. As shown by Rothman (4) and discussed by Hertz (5), in vivo about $85 \%$ of the glutamate cycles in the glutamateglutamine cycle but $15 \%$ is oxidized. Oxidation may take place in astrocytes and neurons, and under basal conditions it does not appear to require glutamate dehydrogenase $(\mathrm{GDH})$, a mitochondrial enzyme present mainly in astrocytes, as a brain-specific-disruption of the gene has small effects on brain glutamate levels (6). De novo synthesis of glutamate takes place in astrocytes thanks to pyruvate carboxylase (PC) $(7,8)$, which is expressed in astrocytes but not in neurons (9-11). This process generates a new molecule of oxaloacetate, which may condense with acetyl-CoA to provide net synthesis of $\alpha$-ketoglutarate, from which glutamate can be formed by transamination (12). Subsequently, glutamine will be synthesized from glutamate via glutamine synthetase, which is exclusively present in the cytosol of astrocytes $(13,14)$. However, this general picture of the glutamate-glutamine cycle may be oversimplified, and there may be important variations, at least, during postnatal development. Recent metabolic studies in the neonatal rat brain have shown that the flow of glutamate from neurons to astrocytes is negligible when compared to the adult brain (15), while, paradoxically, the flow of glutamine from astrocytes to neurons is the same or even larger than in adults. This may suggest that astrocytes are slowly supplying glutamine to neurons so as to build up the neuronal pools of glutamate, aspartate, and $\mathrm{N}$-acetylaspartate (NAA), which are lower than in the adult brain (15), and also, that a molecule other than glutamate is being transferred from neurons to astrocytes.

\section{BRAIN ASPARTATE,} N-ACETYLASPARTATE, GLUTAMINE AND GLUTAMATE LEVELS, AND THE SYNTHESIS OF GLUTAMINE IN VIVO ARE REDUCED IN ARALAR-DEFICIENT MICE

Aralar/AGC1/Slc2512 is an isoform of the mitochondrial carrier of aspartate-glutamate (AGC), a component of the malate-aspartate shuttle (MAS) and it is regulated by calcium from the external side of the inner mitochondrial membrane (16). MAS activity allows the reoxidation of cytosolic NADH which is required to maintain glycolysis coupled to pyruvate oxidation in mitochondria rather than lactate formation. Cytosolic calcium sensed by EF-hands in the N-terminal extensions of aralar carrier regulates MAS activity in neurons (17) and $\beta$-cells (18) and $\mathrm{Ca}^{2+}$ activated respiration in intact neurons (19).
The deficiency in aralar results in a drastic fall in brain aspartate and NAA levels, in a fall in NAA production by neurons in culture, and global hypomyelination both in mice (20-22) and humans (23). Hypomyelination has been attributed to a lack of neuronal NAA which, after transaxonal transport and cleavage by aspartoacylase, is required to supply acetyl groups to developing oligodendrocytes for myelin lipid synthesis $(20,24)$, energy metabolism (25), or other essential functions [reviewed in Ref. (22)].

Aralar deficiency also results in a large fall in brain glutamine content but only a modest decrease in brain glutamate levels, which is not associated to decreases in neuronal glutamate content or changes in synaptosomal glutamate release (26). None of the activities of the three main enzymes involved in the glutamine metabolism [phosphate-activated glutaminase, GDH, and mitochondrial aspartate aminotransferase $(\mathrm{mAST})]$ are changed in the brain of the aralar-KO mice (26). The synthesis of glutamate and glutamine in vivo was also analyzed in aralar-deficient mice at P18-20 after labeling with $\left(1,2-{ }^{13} \mathrm{C}\right)$ acetate or $\left(1-{ }^{13} \mathrm{C}\right)$ glucose. Interestingly, labeled glutamine in the brain of aralar-KO mice was found to be below detection limit, regardless of whether $\left({ }^{13} \mathrm{C}_{2}\right)$ acetate or $\left(1-{ }^{13} \mathrm{C}\right)$ glucose were used as cerebral substrates. These results clearly pointed out to a role for neuronal aralar in glial glutamine production in mouse brain.

\section{EXPRESSION AND FUNCTIONAL RELEVANCE OF ARALAR IN BRAIN CELLS}

A decrease in brain glutamine synthesis in aralar-KO mice is puzzling, in the face of the prominent expression of aralar in neurons rather than in brain astrocytes 
(26-30). This preferential expression in neurons was surprising, as aralar, and also citrin/AGC2/Slc25a13, is definitely present in cultured astroglia $(26,27,31)$ and aralar mRNA has been reported to be present in brain astrocytes $(31,32)$. However, whether aralar is actually used in astrocytes in culture is uncertain, as the lack of aralar in cultured astrocytes did not cause the metabolic alterations characteristic of its deficiency in neurons [increased utilization of external pyruvate, decrease in aspartate levels; (26)]. So far, the most accurate determination of the localization of aralar in brain was that obtained by electron microscopy and immunogold-particle labeling of neuronal and astrocytic mitochondria, especially as the specificity of the labeling was contrasted with the use of aralar-KO mouse brains. These studies showed that neurons contained $94 \%$ of the labeled profiles whereas glial cells (both astrocytes and oligodendrocytes) contained only about $7 \%$, close to the background level (26). These data clearly indicate that, at the protein level, aralar is localized preferentially, if not exclusively, in neurons.

\section{ROLE OF ASPARTATE IN GLUTAMINE SYNTHESIS BY ASTROCYTES}

The most striking defect in the aralardeficient neuron is the complete loss of aspartate. In addition, the levels of other amino acids which give rise to pyruvate also decrease, in response to a failure of MAS which results in a limitation in pyruvate supply to mitochondria. As the defect in glutamine synthesis is necessarily astrocytic, a metabolic limitation in the synthesis of glutamate and glutamine was suspected to happen in brain astrocytes. As the levels of $\alpha$-KG were also maintained in the KO brains, the drop in glial glutamate-glutamine synthesis was attributed to a shortage of the amino-group donor (26). It is known that the de novo glutamate and glutamine production in astrocytes requires the supply of one or two ammonia groups, respectively, and neurons are thought to supply one or both (33). This would depend on whether glutamate oxidation occurs in astrocytes, which would maintain the amino group of glutamate in the astroglial cell, or in neurons, which would not. In this case, neurons would need to supply two amino groups to neighboring astrocytes per newly made glutamine.
It is currently believed that most of the glutamate oxidation occurs in astrocytes (4). Indeed, photoreceptors in the retina avoid the oxidation of glutamate through a tight regulation of the fate of $\alpha-K G$ exerted by the $\mathrm{NAD}^{+} / \mathrm{NADH}$ ratio (34). However, glutamate is also oxidized in photoreceptors and neurons. For example, under hypoglycemia glutamate oxidation takes place through the truncated tricarboxylic acid cycle and aspartate becomes the end product (35-38). The relative importance of astrocytes versus neurons in the oxidation of glutamate in the intact brain both at rest and in conditions of activation is still unknown.

Astrocytes take up aspartate through the same plasma membrane transporters that take up glutamate and with the same affinity (39). In astrocytes, ${ }^{15} \mathrm{~N}$-aspartate is rapidly taken up from the medium (40, 41 ), and its amino group is transferred to glutamate in the aspartate aminotransferase reaction, which then gives rise to glutamine. The fate of the carbon skeleton of aspartate in astrocytes has been also addressed in metabolic studies with $\mathrm{U}_{-}{ }^{13} \mathrm{C}$-aspartate. The results indicate that after an initial transamination, to give rise to oxaloacetate and glutamate, oxaloacetate enters the TCA and is largely incorporated into glutamine. Another large fraction of oxaloacetate is converted into lactate through pyruvate recycling (42-44). These facts suggested that aspartate produced by neurons may be required for glutamate and glutamine production in astrocytes, at least during postnatal development.

To test the role of aspartate in astrocytic glutamate and glutamine synthesis, astrocytes were incubated in the presence of glucose and different amino acids. The supply of 50-200 $\mu \mathrm{M}$ aspartate during $1 \mathrm{~h}$ to astroglial cultures resulted in glutamate and glutamine levels significantly higher than those obtained with all the other amino acids tested including alanine, BCAAs (leucine), and GABA, which, in fact, did not enhance glutamate or glutamine synthesis. Of note, this effect of external aspartate was identical in astrocytes derived from wild type or aralar-KO mice. These findings showed that aspartate is the main amino-group donor for de novo glutamate synthesis in astrocytes and explain why the lack of aspartate produced in neurons causes an impairment in the synthesis of glutamine in aralar-KO mice (26). On the basis of these results we proposed a transcellular traffic of aspartate from neurons to astrocytes which is summarized in Figure 1.

L-Aspartate was proposed to act as a neurotransmitter (46), but this hypothesis has been largely abandoned. However L-aspartate can be taken up in synaptic vesicles and exocytotically released form nerve terminals (47). Whether this is the mechanism utilized for the transcellular flux of aspartate from neurons is unknown. Another possibility for the traffic of aspartate from neurons to astrocytes may involve NAA, as the neuronal transporters of NAA are well established, as is its uptake by astrocytes and subsequent cleavage by aspartoacylase to release acetate and aspartate [reviewed in Ref. (22)].

The neuron-to-astrocyte aspartate efflux pathway described in Figure 1 may provide a means to transfer $\mathrm{NADH} / \mathrm{NAD}^{+}$ redox potential to astrocyte mitochondria an alternative transcellular shuttle system. Indeed, aspartate uptake coupled to OAA production provides a substrate for cytosolic malate dehydrogenase resulting in $\mathrm{NADH}$ consumption in the cytosol and malate formation. As the $\alpha$-ketoglutaratemalate carrier is equally represented in neuronal and glial mitochondria (28), aspartate utilization in glutamate formation in astrocytes will be stoichiometrically related to reducing equivalent transfer to mitochondria. In this way, transcellular aspartate traffic would result in malate oxidation by astrocytic mitochondria. Malate formed in astroglial cytosol may be also transferred back to neurons, as malate is released to a higher extent from cultured astrocytes than from cultured neurons (45).

Alternatively, $\operatorname{Hertz}(5,48)$ has suggested that aralar/AGC1 in brain astrocytes, even at very low levels, could play a role in a modified aspartate-malate shuttle to oxidize reducing equivalents in mitochondria. In this modified shuttle OGC and AGC1 are involved in two different functions: the OGC, in glutamate formation (as shown in Figure 1) and the AGC1 in glutamate oxidation.

\section{CONCLUDING REMARKS}

The role of neuronal aspartate as precursor of glutamate synthesis in astrocytes explains the alterations found in the brains of aralar/ 


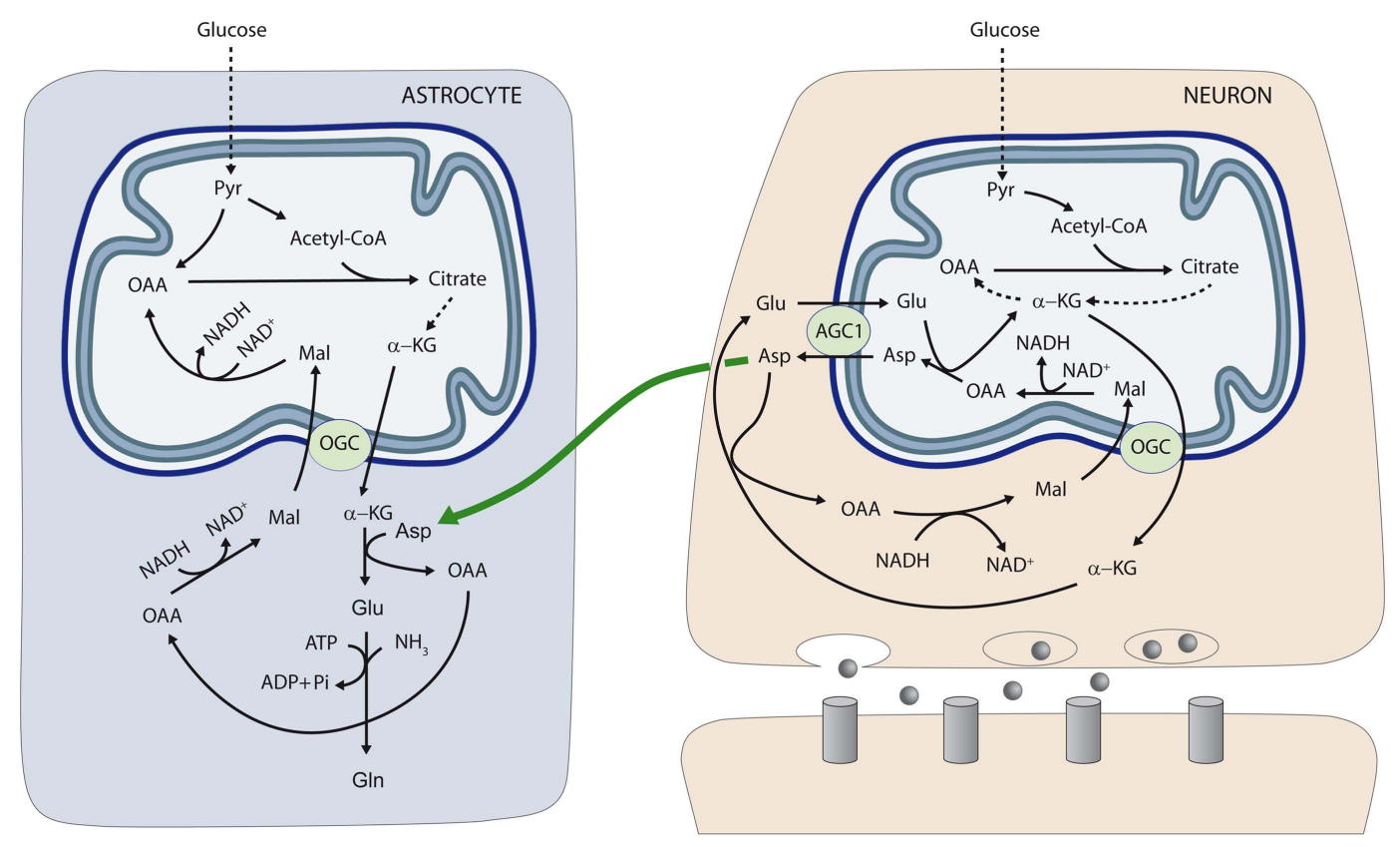

FIGURE 1 | Neuron-to-glia transcellular aspartate efflux pathway for glial glutamate synthesis. Neuronal mitochondria are provided with aralar/AGC1/ SIc25a12 and the oxoglutarate carrier/OGC/SIc25a11 and carry out the malate-aspartate shuttle to transfer NADH reducing equivalents to the mitochondrial matrix. AGC1 is irreversible in polarized mitochondria and the main pathway of glutamate supply to the mitochondrial matrix. As CAST functions in the direction of glutamate formation in cells with an active MAS, mitochondria are the only site where aspartate is produced (in the mitochondrial aspartate aminotransferase reaction), and aspartate leaves the matrix through AGC1 to reach the cytosol. De novo glutamate synthesis in astroglial cells takes place in the cytosol in the CAST reaction with aspartate as amino-nitrogen donor to $\alpha$-KG. A second amino group (possibly arising from ammonia itself formed in neurons in the phosphate-activated glutaminase reaction, or imported from the blood stream) is acquired in the glutamine synthetase reaction and glial glutamine is now transferred to neurons along the glutamate-glutamine cycle (not shown). Oxaloacetate (OAA) arising from the cAST reaction is converted to malate, and malate entry in glial mitochondria along the OGC provides an alternative pathway for redox transfer to mitochondria, which partly compensates for the lack of a malate-aspartate shuttle in brain astrocytes. In this way, equivalent transfer to astroglial mitochondria is stoichiometrically related to de novo glutamate production. Alternatively, malate formed in astroglial cytosol may be transferred back to neurons, as malate is released to a higher extent from cultured astrocytes than from cultured neurons (45) (not shown). The diagram does not address the regulation of the two fates of aspartate in the neuron, MAS (as depicted) or the transfer to the astrocyte (as also depicted), which are obviously mutually exclusive. Thus, transfer of aspartate to astrocytes is associated with glutamate oxidation in neurons, possibly through a truncated TCA cycle, rather than the operation of MAS. AGC, aspartate-glutamate carrier; Asp, aspartate; GIn, glutamine; Glu, glutamate; $\alpha-K G$, $\alpha$-ketoglutarate; Mal, malate; OAA, oxaloacetic acid; OGC, $\alpha$-ketoglutarate-malate carrier; Pyr, pyruvate. Gray circles at presynaptic neuron represent neurotransmitter released; and gray columns at postsynaptic neuron the corresponding receptors [reproduced from Ref. (26)].
AGC1 KO mice, specifically, the impressive drop in the synthesis of glutamine. However, these findings were obtained at $18-20$ days postnatal, at a time where the full development of the glutamate/glutamine cycle has not yet taken place. Further studies in adults will be required to fully understand the role of this transcellular pathway.

\section{REFERENCES}

1. van den Berg CJ, Garfinkel D. A stimulation study of brain compartments. Metabolism of glutamate and related substances in mouse brain. Biochem $J$ (1971) 123:211-8.

2. Gegelashvili G, Schousboe A. High affinity glutamate transporters: regulation of expression and activity. Mol Pharmacol (1997) 52:6-15.

3. Gegelashvili G, Schousboe A. Cellular distribution and kinetic properties of high-affinity glutamate transporters. Brain Res Bull (1998) 45:233-8. doi: 10.1016/S0361-9230(97)00417-6
4. Rothman DL, De Feyter HM, De Graaf RA, Mason GF, Behar KL. 13C MRS studies of neuroenergetics and neurotransmitter cycling in humans. NMR Biomed (2011) 24:943-57. doi: 10.1002/nbm. 1772

5. Hertz L. The glutamate-glutamine (GABA) cycle: importance of late postnatal development and potential reciprocal interactions between biosynthesis and degradation. Front Endocrinol (Lausanne) (2013) 4:59. doi: 10.3389/ fendo.2013.00059

6. Frigerio F, Karaca M, De Roo M, Mlynarik V, Skytt DM, Carobbio S, et al. Deletion of glutamate dehydrogenase 1 (Glud1) in the central nervous system affects glutamate handling without altering synaptic transmission. J Neurochem (2012) 123:342-8. doi: 10.1111/j.1471-4159.2012.07933.x

7. Patel MS. The effect of ketone bodies on pyruvate carboxylation by rat brain mitochondria. $J$ Neurochem (1974) 23:865-7. doi: 10.1111/j.14714159.1974.tb04415.x

8. Waagepetersen HS, Qu H, Schousboe A, Sonnewald U. Elucidation of the quantitative significance of pyruvate carboxylation in cultured cerebellar neurons and astrocytes. J Neurosci Res (2001) 66:763-70. doi: 10.1002/jnr.10061

9. Yu AC, Drejer J, Hertz L, Schousboe A. Pyruvate carboxylase activity in primary cultures of astrocytes and neurons. J Neurochem (1983) 41:1484-7. doi: 10.1111/j.1471-4159.1983.tb00849.x

10. Shank RP, Bennett GS, Freytag SO, Campbell GL. Pyruvate carboxylase: an astrocyte-specific enzyme implicated in the replenishment of amino acid neurotransmitter pools. Brain Res (1985) 329:364-7. doi: 10.1016/0006-8993(85)90552-9

11. Cesar M, Hamprecht B. Immunocytochemical examination of neural rat and mouse primary cultures using monoclonal antibodies raised against pyruvate carboxylase. JNeurochem (1995) 64:23128. doi: 10.1046/j.1471-4159.1995.64052312.x

12. Westergaard N, Drejer J, Schousboe A, Sonnewald U. Evaluation of the importance of transamination versus deamination in astrocytic metabolism of [U-13C]glutamate. Glia (1996) 17:160-8. doi: 10.1002/(SICI)1098-1136(199606)17:2<160::AIDGLIA7>3.3.CO;2-S 
13. Martinez-Hernandez A, Bell KP, Norenberg MD. Glutamine synthetase: glial localization in brain. Science (1977) 195:1356-8. doi: 10.1126/ science. 14400

14. Norenberg MD, Martinez-Hernandez A. Fine structural localization of glutamine synthetase in astrocytes of rat brain. Brain Res (1979) 161:303-10. doi: 10.1016/0006-8993(79)90071-4

15. MorkenTS, BrekkeE,HabergA, Wideroe M, Brubakk AM, Sonnewald U. Neuron-astrocyte interactions, pyruvate carboxylation and the pentose phosphate pathway in the neonatal rat brain. Neurochem Res (2013). doi: 10.1007/s11064-013-1014-3

16. Palmieri L, Pardo B, Lasorsa FM, Del Arco A, Kobayashi K, Iijima M, et al. Citrin and aralarl are $\mathrm{Ca}(2+)$-stimulated aspartate/glutamate transporters in mitochondria. EMBOJ (2001) 20:5060-9. doi: 10.1093/emboj/20.18.5060

17. Pardo B, Contreras L, Serrano A, Ramos M, Kobayashi K, Iijima M, et al. Essential role of aralar in the transduction of small Ca2+ signals to neuronal mitochondria. J Biol Chem (2006) 281:103947. doi: 10.1074/jbc.M507270200

18. Marmol P, Pardo B, Wiederkehr A, Del Arco A, Wollheim CB, Satrustegui J. Requirement for aralar and its $\mathrm{Ca} 2+$-binding sites in $\mathrm{Ca} 2+$ signal transduction in mitochondria from INS-1 clonal beta-cells. J Biol Chem (2009) 284:515-24. doi: 10.1074/jbc. M806729200

19. Llorente-Folch I, Rueda CB, Amigo I, Del Arco A, Saheki T, Pardo B, et al. Calcium-regulation of mitochondrial respiration maintains ATP homeostasis and requires ARALAR/AGC1malate aspartate shuttle in intact cortical neurons. J Neurosci (2013) 33:13957-71. doi: 10.1523/ JNEUROSCI.0929-13.2013

20. Jalil MA, Begum L, Contreras L, Pardo B, Iijima M, Li MX, et al. Reduced $\mathrm{N}$-acetylaspartate levels in mice lacking aralar, a brain- and muscle-type mitochondrial aspartate-glutamate carrier. J Biol Chem (2005) 280:31333-9. doi: 10.1074/jbc.M505286200

21. Sakurai T, Ramoz N, Barreto M, Gazdoiu M, Takahashi N, Gertner M, et al. Slc25a12 disruption alters myelination and neurofilaments: a model for a hypomyelination syndrome and childhood neurodevelopmental disorders. Biol Psychiatry (2010) 67:887-94. doi: 10.1016/j.biopsych.2009.08.042

22. Ramos M, Pardo B, Llorente-Folch I, Saheki T, Del Arco A, Satrustegui J. Deficiency of the mitochondrial transporter of aspartate/glutamate aralar/AGC1 causes hypomyelination and neuronal defects unrelated to myelin deficits in mouse brain. J Neurosci Res (2011) 89:2008-17. doi: 10.1002/jnr.22639

23. Wibom R, Lasorsa FM, Tohonen V, Barbaro M, Sterky FH, Kucinski T, et al. AGC1 deficiency associated with global cerebral hypomyelination. N Engl J Med (2009) 361:489-95. doi: 10.1056/ NEJMoa0900591

24. Satrustegui J, Contreras L, Ramos M, Marmol P, Del Arco A, Saheki T, et al. Role of aralar, the mitochondrial transporter of aspartate-glutamate, in brain $\mathrm{N}$-acetylaspartate formation and $\mathrm{Ca}(2+)$ signaling in neuronal mitochondria. J Neurosci Res (2007) 85:3359-66. doi: 10.1002/jnr.21299

25. Francis JS, Strande L, Markov V, Leone P. Aspartoacylase supports oxidative energy metabolism during myelination. JCereb Blood Flow Metab (2012) 32:1725-36. doi: 10.1038/jcbfm.2012.66
26. Pardo B, Rodrigues TB, Contreras L, Garzon M, Llorente-Folch I, Kobayashi K, et al. Brain glutamine synthesis requires neuronal-born aspartate as amino donor for glial glutamate formation. $J$ Cereb Blood Flow Metab (2011) 31:90-101. doi: 10.1038/jcbfm.2010.146

27. Ramos M, Del Arco A, Pardo B, Martinez-Serrano A, Martinez-Morales JR, Kobayashi K, et al. Developmental changes in the Ca2+-regulated mitochondrial aspartate-glutamate carrier aralar1 in brain and prominent expression in the spinal cord. Brain Res Dev Brain Res (2003) 143:33-46. doi: 10.1016/S0165-3806(03)00097-X

28. Berkich DA, Ola MS, Cole J, Sweatt AJ, Hutson SM, Lanoue KF. Mitochondrial transport proteins of the brain. J Neurosci Res (2007) 85:3367-77. doi: 10.1002/jnr.21500

29. Xu Y, Ola MS, Berkich DA, Gardner TW, Barber AJ, Palmieri F, et al. Energy sources for glutamate neurotransmission in the retina: absence of the aspartate/glutamate carrier produces reliance on glycolysis in glia. J Neurochem (2007) 101:120-31. doi: 10.1111/j.1471-4159.2006.04349.x

30. Cahoy JD, Emery B, Kaushal A, Foo LC, Zamanian JL, Christopherson KS, et al. A transcriptome database for astrocytes, neurons, and oligodendrocytes: a new resource for understanding brain development and function. J Neurosci (2008) 28:264-78. doi: 10.1523/JNEUROSCI.4178-07.2008

31. Li B, Hertz L, Peng L. Aralar mRNA and protein levels in neurons and astrocytes freshly isolated from young and adult mouse brain and in maturing cultured astrocytes. Neurochem Int (2012) 61:1325-32. doi: 10.1016/j.neuint.2012.09.009

32. Lovatt D, Sonnewald U, Waagepetersen HS, Schousboe A, He W, Lin JH, et al. The transcriptome and metabolic gene signature of protoplasmic astrocytes in the adult murine cortex. J Neurosci (2007) 27:12255-66. doi: 10.1523/ JNEUROSCI.3404-07.2007

33. Bak LK, Schousboe A, Waagepetersen HS. The glutamate/GABA-glutamine cycle: aspects of transport, neurotransmitter homeostasis and ammonia transfer. J Neurochem (2006) 98:641-53. doi: 10.1111/j.1471-4159.2006.03913.x

34. Du J, Cleghorn W, Contreras L, Linton J, Chan G, Chertov A, et al. Cytosolic reducing power preserves glutamate in retina. Proc Natl Acad Sci U S A (Forthcoming).

35. Yudkoff M, Nelson D, Daikhin Y, Erecinska M. Tricarboxylic acid cycle in rat brain synaptosomes. Fluxes and interactions with aspartate aminotransferase and malate/aspartate shuttle. J Biol Chem (1994) 269:27414-20.

36. Honegger $\mathrm{P}$, Braissant $\mathrm{O}$, Henry $\mathrm{H}$, Boulat $\mathrm{O}$, Bachmann C, Zurich MG, et al. Alteration of amino acid metabolism in neuronal aggregate cultures exposed to hypoglycaemic conditions. J Neurochem (2002) 81:1141-51. doi: 10.1046/j.1471-4159.2002.00888.x

37. Amaral AI. Effects of hypoglycaemia on neuronal metabolism in the adult brain: role of alternative substrates to glucose. J Inherit Metab Dis (2013) 36:621-34. doi: 10.1007/s10545-012-9553-3

38. Liu K, Ye XJ, Hu WY, Zhang GY, Bai GH, Zhao LC, et al. Neurochemical changes in the rat occipital cortex and hippocampus after repetitive and profound hypoglycemia during the neonatal period: an ex vivo
H Magnetic Resonance Spectroscopy Study. Mol Neurobiol (2013). doi: 10.1007/s12035-013-8446-2

39. Rao VL, Murthy CR. Uptake and metabolism of glutamate and aspartate by astroglial and neuronal preparations of rat cerebellum. Neurochem Res (1993) 18:647-54. doi: 10.1007/BF00966777

40. Yudkoff M, Nissim I, Hummeler K, Medow M, Pleasure D. Utilization of [15N]glutamate by cultured astrocytes. Biochem J (1986) 234:185-92.

41. Erecinska M, Pleasure D, Nelson D, Nissim I, Yudkoff M. Cerebral aspartate utilization: near-equilibrium relationships in aspartate aminotransferase reaction. JNeurochem (1993) 60:1696-706. doi: 10.1111/ j.1471-4159.1993.tb13393.x

42. Bakken IJ, White LR, Aasly J, Unsgard G, Sonnewald U. Lactate formation from [U-13C] aspartate in cultured astrocytes: compartmentation of pyruvate metabolism. Neurosci Lett (1997) 237:117-20. doi: 10.1016/S0304-3940(97)00834-3

43. Bakken IJ, White LR, Aasly J, Unsgard G, Sonnewald U. [U-13C] aspartate metabolism in cultured cortical astrocytes and cerebellar granule neurons studied by NMR spectroscopy. Glia (1998) 23:271-7. doi: 10.1002/(SICI) 1098-1136(199807)23:3<271::AIDGLIA9 $>3.0 . C O ; 2-7$

44. Murin R, Mohammadi G, Kowtharapu BS, Leibfritz D, Hamprecht B. Metabolism of [U-(13)C] aspartate by astroglial cultures: nuclear magnetic resonance analysis of the culture media. Neurochem Res (2010) 35:2053-61. doi: 10.1007/s11064-010-0326-9

45. Westergaard N, Sonnewald U, Schousboe A. Release of alpha-ketoglutarate, malate and succinate from cultured astrocytes: possible role in amino acid neurotransmitter homeostasis. Neurosci Lett (1994) 176:105-9. doi: 10.1016/0304-3940(94)90882-6

46. Nadler JV, Vaca KW, White WF, Lynch GS, Cotman CW. Aspartate and glutamate as possible transmitters of excitatory hippocampal afferents. Nature (1976) 260:538-40. doi: 10.1038/260538a0

47. Morland C, Nordengen K, Larsson M, Prolo LM, Farzampour Z, Reimer RJ, et al. Vesicular uptake and exocytosis of L-aspartate is independent of sialin. FASEB J (2013) 27:1264-74. doi: 10.1096/ f. $12-206300$

48. HertzL. Brain glutamine synthesis requires neuronal aspartate: a commentary. J Cereb Blood Flow Metab (2011) 31:384-7. doi: 10.1038/jcbfm.2010.199

Received: 30 September 2013; accepted: 02 October 2013; published online: 15 October 2013.

Citation: Pardo B, Contreras L and Satrústegui J (2013) De novo synthesis of glial glutamate and glutamine in young mice requires aspartate provided by the neuronal mitochondrial aspartate-glutamate carrier aralar/AGC1. Front. Endocrinol. 4:149. doi: 10.3389/ fendo.2013.00149

This article was submitted to Cellular Endocrinology, a section of the journal Frontiers in Endocrinology.

Copyright $\odot 2013$ Pardo, Contreras and Satrústegui. This is an open-access article distributed under the terms of the Creative Commons Attribution License (CC BY). The use, distribution or reproduction in other forums is permitted, provided the original author(s) or licensor are credited and that the original publication in this journal is cited, in accordance with accepted academic practice. No use, distribution or reproduction is permitted which does not comply with these terms. 